\title{
CT Enterography Using Four Different Endoluminal Contrast Agents: A Comparative Study
}

\author{
Deepak Singla ${ }^{1, \odot}$ Shruti Chandak ${ }^{1} \quad$ Ankur Malhotra $^{1}$ Arjit Agarwal ${ }^{1}$ \\ Tanu Raman ${ }^{1}$ Mohini Chaudhary ${ }^{1}$ \\ ${ }^{1}$ Department of Radiodiagnosis, Teerthanker Mahaveer Medical \\ College and Research Centre, Moradabad, Uttar Pradesh, India

\begin{abstract}
Address for correspondence Shruti Chandak, MBBS, MD, Department of Radiodiagnosis, Teerthanker Mahaveer Medical College and Research Centre, Moradabad, Uttar Pradesh 244001, India (e mail: chandakshruti@yahoo.com).
\end{abstract}

J Gastrointestinal Abdominal Radiol ISGAR 2022;5:16-22

\begin{abstract}
Keywords

- intestine

- iohexol

- mannitol

- multidetector computed tomography

- polyethylene glycols

- radiology

Objectives To determine the most preferable endoluminal contrast agent among mannitol, polyethylene glycol (PEG), iohexol, and water by comparing various qualitative (distension, fold visibility, and homogeneity) and quantitative parameters (distension) along with artifacts and patient feedback for computed tomography enterography (CTE).

Methods This was a prospective study including 120 patients of age more than or equal to 18 years who were randomized equally into four groups. Group 1 was given $1500 \mathrm{~mL}$ of $3 \%$ mannitol solution, group 2 was given $1500 \mathrm{~mL}$ of PEG, group 3 was given $20 \mathrm{~mL}$ of iohexol dissolved in $1500 \mathrm{~mL}$ of water, and group 4 was given $1500 \mathrm{~mL}$ of plain water. CTE was done and images were evaluated in axial and coronal planes. Various quantitative and qualitative parameters were taken at the level of second part of duodenum, jejunum, ileum and ileocecal junction (ICJ). Artifacts and patient feedback were also taken into consideration.

Results The quantitative distension and grading, qualitative distension, fold visibility, and homogeneity of the second part of duodenum, jejunum at the level of superior mesenteric artery, inferior mesenteric artery and renal artery on both sides of abdomen, ileum at the level of aortic bifurcation, common iliac bifurcation, and deep pelvis on both sides of abdomen and IC] were significantly more in PEG group as compared with mannitol group, followed by iohexol and water group. The results were calculated by ANOVA test using $p$-value. In terms of patient feedback and artifacts, water was the best agent.

Conclusions PEG is the most suitable contrast agent to carry out CTE. Distension, fold visibility, and homogeneity are the essential features for a better diagnostic outcome of CTE, which was better with PEG.
\end{abstract}

\section{Introduction}

The small intestine has always been a challenging area for both physicians as well as surgeons, owing to the entirety of

published online June 11, 2021
DOI https://doi.org/ $10.1055 / \mathrm{s}-0041-1730101$ ISSN 2581-9933 its length as well as the complexity of its nature. In the past, imaging of small intestine was done by conducting gut transit time tests or barium studies that used to yield nonspecific results, but nowadays "CT enterography (CTE) and MR
(C) 2021. Indian Society of Gastrointestinal and Abdominal Radiology This is an open access article published by Thieme under the terms of the Creative Commons Attribution-NonDerivative-NonCommercial-License, permitting copying and reproduction so long as the original work is given appropriate credit. Contents may not be used for commercial purposes, or adapted, remixed, transformed or built upon. (https://creativecommons.org/licenses/by-nc-nd/4.0/).

Thieme Medical and Scientific Publishers Pvt. Ltd. A-12, 2nd Floor, Sector 2, Noida-201301 UP, India 
enterography (MRE)" are the main imaging modalities used to look for small bowel pathologies. ${ }^{1,2}$ Further advancements in multidetector CT (MDCT) have transformed the imaging of solid and hollow viscera. ${ }^{1}$ Other imaging modalities include $\mathrm{CT}$, MR, fluoroscopic enteroclysis, and capsule endoscopy. ${ }^{2}$

CTE and MRE both are equally useful in the imaging of small bowel pathologies. MRE has a major advantage of being a radiation-free imaging modality, which is a beneficial point, especially in pediatric and follow-up patients. But MRE has limited availability, is costlier, has a longer acquisition time, and requires sedation, especially in patients of pediatric age group for proper acquisition. CTE uses ionizing radiation but due to its widespread availability, cost effectivity, and time efficiency with higher temporal and spatial resolution, it is a preferable modality in general and especially in acute emergencies without any need for sedation., ${ }^{2,3}$

In CTE, approximately 1300 to $2000 \mathrm{~mL}$ of oral contrast agent (neutral or positive) is administered over 60 minutes in divided increments prior to the scan along with intravenous (IV) administration of 3 to $5 \mathrm{~mL}$ of iodinated contrast agent per second, with imaging during enteric or portal phase. Enteric phase is acquired at 50 seconds after the initiation of IV contrast during which the peak enhancement of small bowel loops is noted. Enteric phase plays an important role in demonstrating the findings of Crohn's disease, small bowel vascular lesions, and enhancing polyps. Synchronous evaluation of pancreas and liver can also be done in enteric phase and portal phase, respectively, if needed. ${ }^{4-6}$

Adequate bowel distention is required for the diagnosis of small bowel diseases, since poorly distended bowel loops may obscure intraluminal pathologies or mimic luminal wall thickening and areas of increased attenuation of bowel wall segments. ${ }^{7-9}$ Rapid water absorption, prolonged gastric emptying, inadequate consumption of oral contrast agent, and image acquisition shortly after consumption of oral contrast agent may result in inadequate distension or luminal collapse. ${ }^{10}$

Oral contrast agents used can be neutral or positive enteric agents. Neutral agents have a CT attenuation number similar to that of water, whereas positive contrast agents have a CT attenuation number more than that of enhancing structures. Neutral contrast agents allow better visualization of hyperenhancing bowel wall as in case of segmental inflammation. Positive contrast agents are helpful in diagnosing intraluminal filling defects and differentiating between bowel loops and extraluminal structures like mesenteric lymph node or tumor. However, with positive contrast agent, mucosal enhancement is masked, which is a crucial finding in differentiation of various small bowel pathologies, and in 3D angiographic reconstruction, with volume rendering or maximum intensity projection (MIP), overlap between contrast-filled bowel loops and contrast-enhanced mesenteric vessels occurs, which is also problematic. ${ }^{4-6}$

Examples of endoluminal contrast agents:

- Neutral agents include water, combination of water and methylcellulose, lactulose, polyethylene glycol (PEG), low concentration $(0.1 \% \mathrm{w} / \mathrm{v})$ barium solution mixed with sorbitol, milk, mannitol, psyllium fiber, and locust bean gum., ${ }^{4,5,11}$
- Positive agents include 1 to $2 \%$ barium sulfate suspension and 2 to $3 \%$ water soluble iodinated solution. ${ }^{12}$

Proper image acquisition and optimal patient preparation are important for precise diagnosis of small bowel pathologies. Luminal distension and mucosal fold visualization are the critical determining factors in gastrointestinal (GI) imaging. Isotropic imaging, multiplanar reconstruction (MPR), techniques of neutral or positive oral contrast agent administration to increase bowel loop distension, in order to provide adequate visualization and delay the absorption of contrast by the bowel through its villi and mucosal surface, apart from image acquisition intended toward maximum bowel wall enhancement time is called CTE.,8

Nowadays, neutral contrast agents are preferred for abdominal and pelvic CT imaging, as they accomplish most of the criteria of an ideal contrast agent. Many neutral oral contrast agents have been avidly studied in literature, namely, "water, water in comparison with methylcellulose, $0.1 \%$ barium solution, sorbitol, polyethylene glycol, mannitol solution, and milk."7-9,13,14

Lower concentration of PEG is used with the purpose to decrease its undesirable effects, while preserving its distension capability. Storage of whole milk is quite difficult, and it may not be acceptable in such large quantity, which causes limitation in its usage. ${ }^{15}$ The poor palatability of methylcellulose solution makes it less preferable, and PEG solution, despite its exceptional capability of distention, is purgative in nature. The most prevalent neutral contrast material, which can be used extensively in the patients that require imaging, is water. ${ }^{16}$ Due to slow gut transit time of water, contrast capacity in the proximal part of the gastrointestinal tract (GIT) is decent but rapid absorption of water in the distal small bowel, especially in ileum, which makes it unfavorable to look for ileal pathologies. ${ }^{6}$

Adding agent with higher osmolality in water might decrease its rate of absorption from the GIT. Sorbitol is one of the neutral and cost-effective additive agents, which can be used in combination with low-density barium (VoLumen) for diagnosing small intestinal disorders. ${ }^{16}$

This study was done to compare bowel distension, homogeneity, and fold visibility among mannitol, PEG, iohexol, and water. Along with these parameters, patient's acceptance is also taken into account.

As of now, many oral contrast agents are available in the market, and multiple studies are being conducted to compare these agents, but to the best of our knowledge, no other study compared these four endoluminal contrast agents.

\section{Materials and Methods}

The CTE was done using 128 slice MDCT (Ingenuity, Philips Imaging System, The Netherlands) by following CTE protocol. Our study was a prospective randomized trial, and it was done on 120 patients who were equally randomized into four groups by the chit and box system. This study was started after approval from the Institutional Ethical Committee and Institutional Review Board. 


\section{Patient Distribution}

Patients were randomized into four groups, and out of four, one group was allocated.

- Group 1 was given $1500 \mathrm{~mL}$ of $3 \%$ mannitol solution (Shandong Tianli Pharmaceutical Co. Ltd., China).

- Group 2 was given PEG (Peglec, Tablets India Limited, Chennai, India) solution in $1500 \mathrm{~mL}$ of water.

- Group 3 was given $20 \mathrm{~mL}$ of iohexol (Omnipaque $300 \mathrm{GE}$ Healthcare, Shanghai, China) in $1500 \mathrm{~mL}$ of water.

- Group 4 was given $1500 \mathrm{~mL}$ of plain water.

Group 1 received 3\% mannitol in water (45 g of mannitol was dissolved in $1500 \mathrm{~mL}$ of water to make a $3 \%$ solution). Group 2 received 137.15 gm of PEG dissolved in $1500 \mathrm{~mL}$ water. Group 3 received positive contrast agent, that is, iohexol (20 mL dissolved in $1500 \mathrm{~mL}$ of water). Group 4 received $1500 \mathrm{~mL}$ of plain water without any additives. All patients were given $1500 \mathrm{~mL}$ of contrast to consume over a period of 45 to 60 minutes; every 4 to 5 minutes, $150 \mathrm{~mL}$ of contrast agent should be consumed for 45 to 55 minutes, and CTE was done 55 to 60 minutes after the initiation of consumption of oral contrast agent. ${ }^{17}$

A total of $20 \mathrm{mg}$ of IV hyoscine was also given immediately prior to CTE. ${ }^{17}$

\section{Scanning Protocol}

CTE was done using 128 slice MDCT (Ingenuity, Philips Imaging System, The Netherlands) from the diaphragm to the pubic symphysis.

Using a power injector, 80 to $100 \mathrm{~mL}$ contrast of concentration $300 \mathrm{mg}$ iodine/mL (Omnipaque $300 \mathrm{GE}$ healthcare, Shanghai, China) was IV administered at an injection rate of 3 to $4 \mathrm{~mL} / \mathrm{sec}$, followed by flushing of $40 \mathrm{~mL}$ normal saline. ${ }^{17}$ Multiphasic studies were performed on the basis of clinical and radiological indications. Bolus tracking method (tracker in lower thoracic aorta) was used for acquisition of both arterial as well as portovenous phases. Arterial phase and portovenous phase were taken with a postthreshold delay of 8 seconds and 45 seconds, respectively. MPR was done, and images were archived in axial and coronal planes, with a slice thickness of $1 \mathrm{~mm}$ and interval of $0.5 \mathrm{~mm}$, and analyzed on CT workstation. ${ }^{1}$

\section{Image Analysis}

Image analysis was done by an experienced radiologist who was blinded to the group of oral contrast agents, that is, mannitol, PEG, iodinated positive contrast, and plain water. Qualitative and quantitative analysis was performed for distension, morphology of bowel (wall enhancement, stratification and luminal contents), and overall image quality by using actual measurements for quantitative analysis and point scale system for qualitative analysis.

\section{Quantitative Analysis}

It was performed by assessing the images in axial section. Two maximally distended bowel loops were taken on both right and left sides at a specific anatomical level, and outer to outer wall diameter was measured. To obtain systemized data, measurements were done at the level of second part of duodenum, superior mesenteric artery, renal artery, inferior mesenteric artery levels for jejunum and aortic, common iliac bifurcation and deep pelvis for ileal loops. Following this, the mean was calculated. Quantitative analysis of bowel wall and fold visibility were performed on axial images at the same levels by using three grades.

Grade 0-collapsed bowel/poor contrast between the wall and intraluminal contents.

Grade 1-endoluminal contrast agent seen within the bowel loops, with distension of $\leq 1.5 \mathrm{~cm}$ and incomplete fold visibility.

Grade 2-distension $>1.5 \mathrm{~cm}$ and complete visibility of fold. ${ }^{17}$

\section{Qualitative Analysis}

Qualitative analysis of distension, homogeneity of luminal contents, and wall visibility were done on a continuous 3-point scale using coronal images.

Score 0 -fair ( $<25 \%$ of the bowel loops show adequate distension, homogeneity of luminal contents, or fold visibility). Score $1-\operatorname{good}$ ( 25 to $<75 \%$ of the bowel loops show adequate distension, homogeneity of luminal contents, or fold visibility).

Score $2-$ excellent ( 75 to $100 \%$ of the bowel loops show adequate distension, homogeneity of luminal contents, or fold visibility). ${ }^{17}$

The grading of artifacts was done in the following way:

Grade $0-$ no artifacts.

Grade 1-artifacts with no degradation of image quality. Grade 2-artifacts causing degradation of image quality.

Ileocecal junction (ICJ) was separately evaluated on coronal planes for distension and mural pattern, using same grades and point scale.

\section{Acceptability}

To check for acceptability and side effects of oral contrast agents, a questionnaire was given to patients consisting of palatability, score ranging from 1 to 3: 1-bad taste; 2-represent reasonable taste; 3 -represent good taste; for nausea, on a scale of 1 to 3: 1-no nausea; 2-mild nausea; 3-severe nausea; and report the episode of diarrhea if present. ${ }^{17}$ The patients were followed up for 1 hour for any contrast-related reactions.

\section{Statistical analysis}

The data was entered in Microsoft Excel sheets (2016 version). Statistical analysis was performed using SPSS software (Version 20.0). Mean and standard deviation (SD) were obtained. The results were found to be statistically significant if the $p$-values were below 0.05 .

\section{Results}

The patients were randomized equally into four group (i.e., each group having 30 patients). In our study demographics, 
there were $55.83 \%$ males and $44.17 \%$ females, and the mean age was $37.63 \pm 15.17$ (highest age was 70 years and lowest was 18 years). The quantitative distension of second part of duodenum ( $p$-value $=0.001)$, jejunum at the level of superior mesenteric artery ( $p$-value on right side and left side 0.001 and $<0.001$, respectively), inferior mesenteric artery ( $p$-value on right side and left side 0.001 and 0.001 , respectively) and renal artery ( $p$-value on right side and left side 0.002 and $<0.001$, respectively) on both sides of abdomen, ileum at the level of aortic bifurcation ( $p$-value on right side and left side 0.001 and 0.001 , respectively), common iliac bifurcation ( $p$-value on right side and left side 0.001 and 0.001 , respectively), and deep pelvis ( $p$-value on right side and left side 0.001 and 0.001 , respectively) on both sides of abdomen and ICJ ( $p$-value $\leq 0.01$ ) were significantly more in PEG group as compared with mannitol group, followed by iohexol and water group (-Figs. 1-4). The mean and mean of maximum distension of second part of duodenum ( $\mathrm{p}$-value $\leq 0.01$ and 0.001 , respectively), jejunum ( $p$-value $\leq 0.01$ and 0.001 , respectively), ileum ( $p$-value $\leq 0.01$ and 0.001 , respectively), and ICJ ( $p$-value $=<0.01$ and 0.001 , respectively) were also highest in PEG group followed by mannitol, iohexol, and water group. In quantitative grading of distension of second part of duodenum, jejunum, ileum and ICJ, PEG group was also maximum at second part of duodenum $(p$-value $=$ $0.004)$, jejunum $(p$-value $=0.001)$ and ICJ $(p$-value $=0.001)$, whereas at ileum ( $p$-value $=0.001)$, all the cases in mannitol as well as PEG group showed grade 2 distension. The results were statistically significant as determined by ANOVA test using $p$-value. In qualitative analysis distension, fold visibility and homogeneity were highest in PEG group, followed by mannitol, iohexol and water group, which was statistically significant ( $p$-value $\leq 0.001)$ (-Fig. 5). Artifacts were noted in one case of PEG group, which was statistically insignificant ( $p$-value $=0.388)$. In terms of palatability, water was reported to be the best, followed by iohexol and mannitol, whereas PEG was reported to be worst, and the difference was statistically significant ( $p$-value $\leq 0.001$ ). Nausea was not reported in any case of water, followed by mannitol group, in which 29 cases reported no nausea and 1 case reported mild nausea; in PEG group, 17 cases reported no nausea and 13 cases reported mild nausea; and in iohexol group, 15 cases reported no nausea, 14 cases reported mild nausea, and 1 case reported severe nausea, which was statistically significant ( $p$-value $\leq$ 0.001). Episodes of vomiting were noted in PEG and iohexol group, whereas no episodes were noted in mannitol and plain water, but it was statistically insignificant ( $p$-value $=0.247$ ). Statistically significant ( $p$-value $\leq 0.001$ ) episodes of diarrhea were noted in eight patients in mannitol group, whereas no episode was noted in rest of the groups.

\section{Discussion}

Small intestine imaging is very challenging due to its long length, caliber, peristaltic movements, and convoluted and compactly packed loops. ${ }^{18}$ With the advent of MDCT technology, spatial and contrast resolution has been improved, which further aids in diagnosis. Further advancement in technology leading to development of MPR, maximum intensity projection (MIP) and volume rendering (VR) images, CTE has become popular investigation for diagnosing small bowel pathologies, as it aids in localization and extent of disease. ${ }^{19}$ For proper evaluation of small bowel and good mural visualization, adequate bowel distension is a must, because collapsed loops can lead to misdiagnosis. Various endoluminal contrast agents are used for achieving adequate bowel

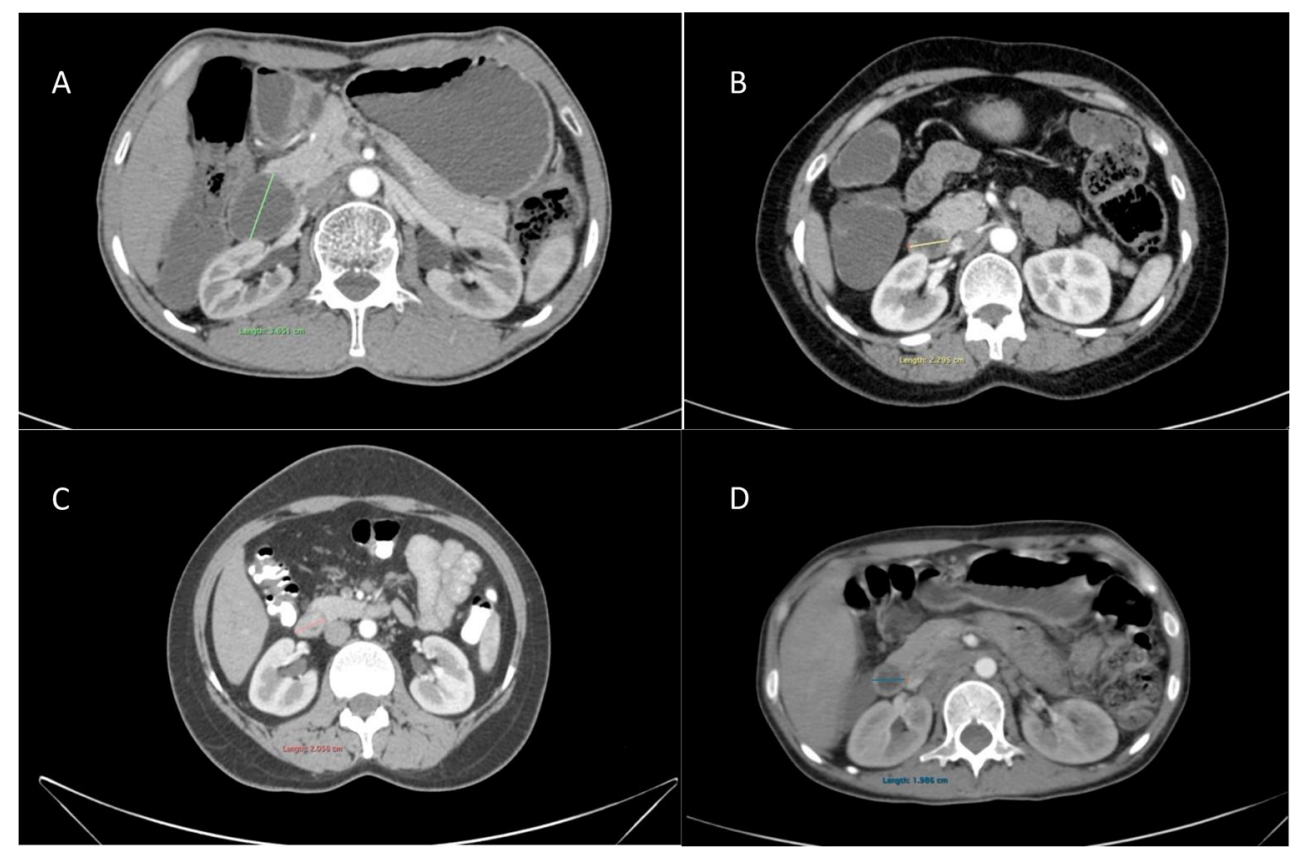

Fig. 1 CT enterography shows variable distension of second part of duodenum with neutral endoluminal contrast agent (A) polyethylene glycol (PEG), (B) mannitol (C) with positive contrast agent, that is, iohexol and (D) with plain water. The distension was maximum with PEG followed by mannitol. 


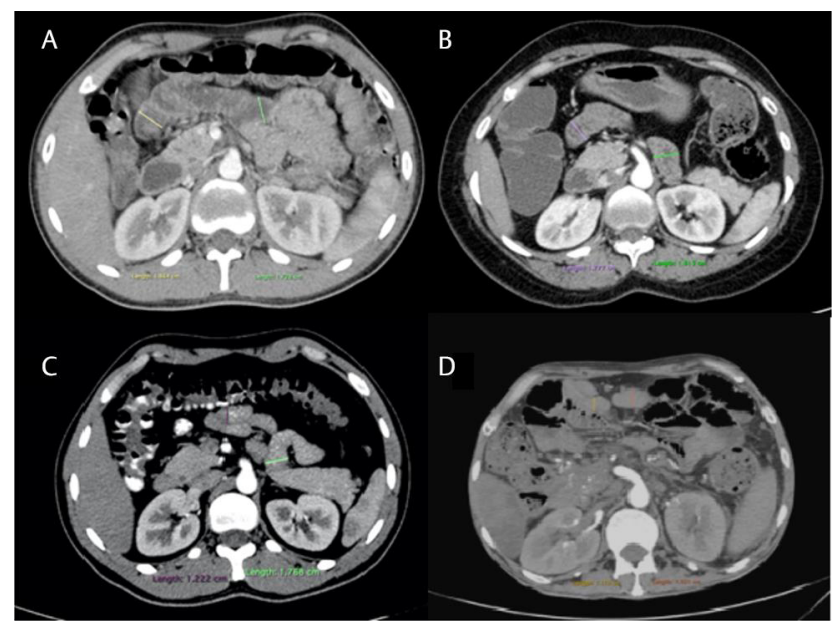

Fig. 2 CT enterography showing distension of jejunum at the level of superior mesenteric artery on right as well as left side (A) with polyethylene glycol (PEG), (B) with mannitol, (C) with iohexol and (D) with plain water.

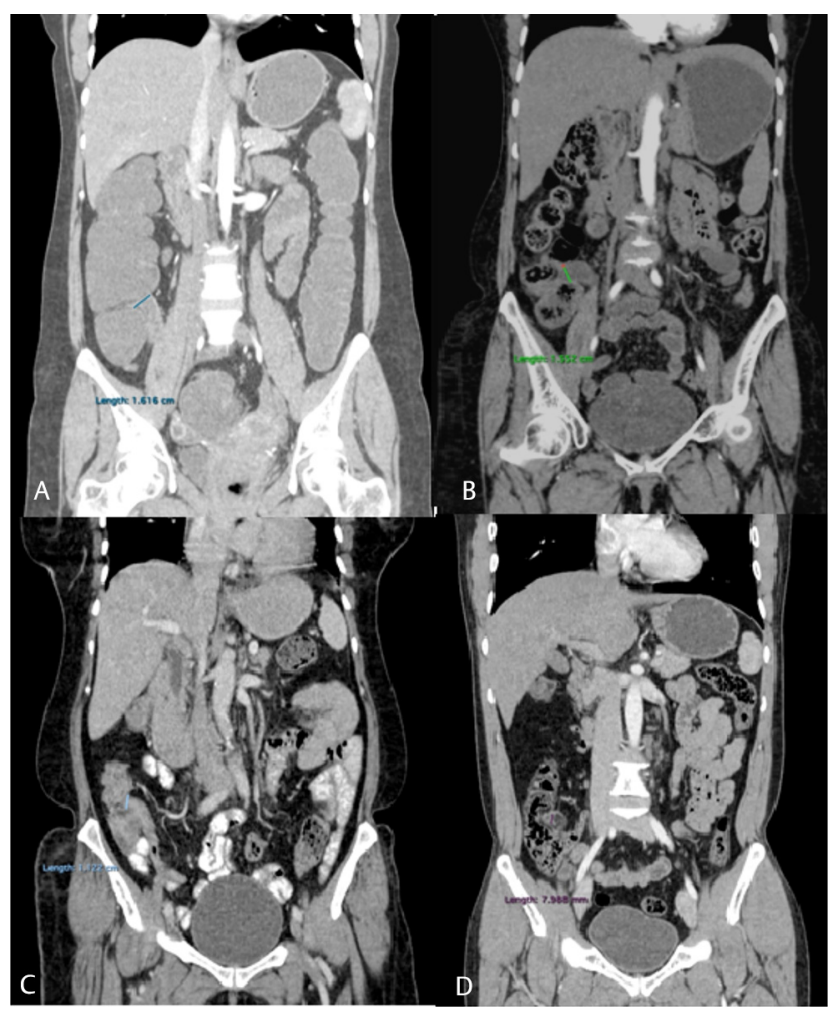

Fig. 4 CT enterography showing distension of ileocecal junction (ICJ) with (A) polyethylene glycol (PEG) (B) mannitol (C) iohexol and (D) plain water.

distension. These endoluminal contrast agents are mainly of two types: neutral and positive. There have been many studies done in the past 30 to 40 years to find out the most suitable contrast agent. In the past, positive contrast agents were the most preferred agents. Positive contrast agents help in differentiating extraluminal and intraluminal fluid collection, and diagnosing intraluminal filling defects and cystic lesions, but the major drawback of positive contrast agent is obscuration of mucosal enhancement pattern due to its high attenuating character, especially in distal ileum which is the

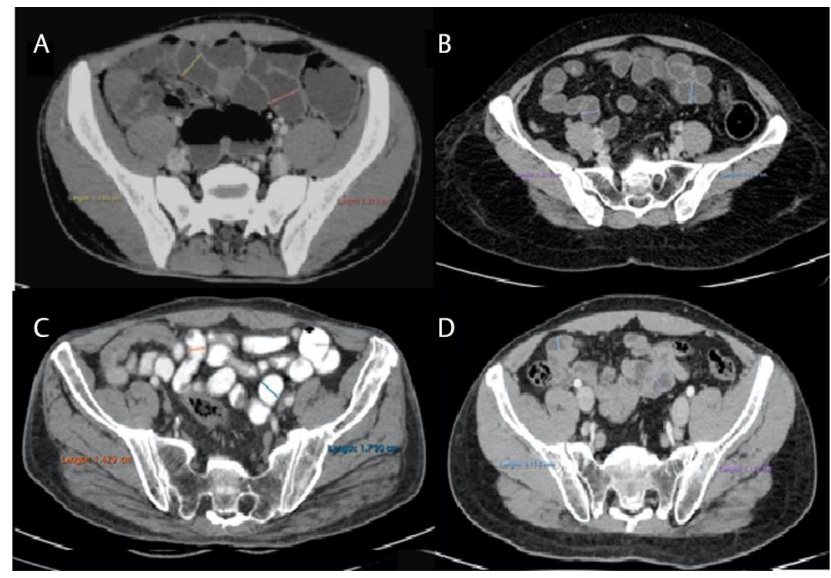

Fig. 3 CT enterography showing distension of ileum in deep pelvis on right as well as left side (A) with polyethylene glycol (PEG) (B) with mannitol (C) with iohexol and (D) with plain water. The distension of ileum was maximum with PEG followed by mannitol.

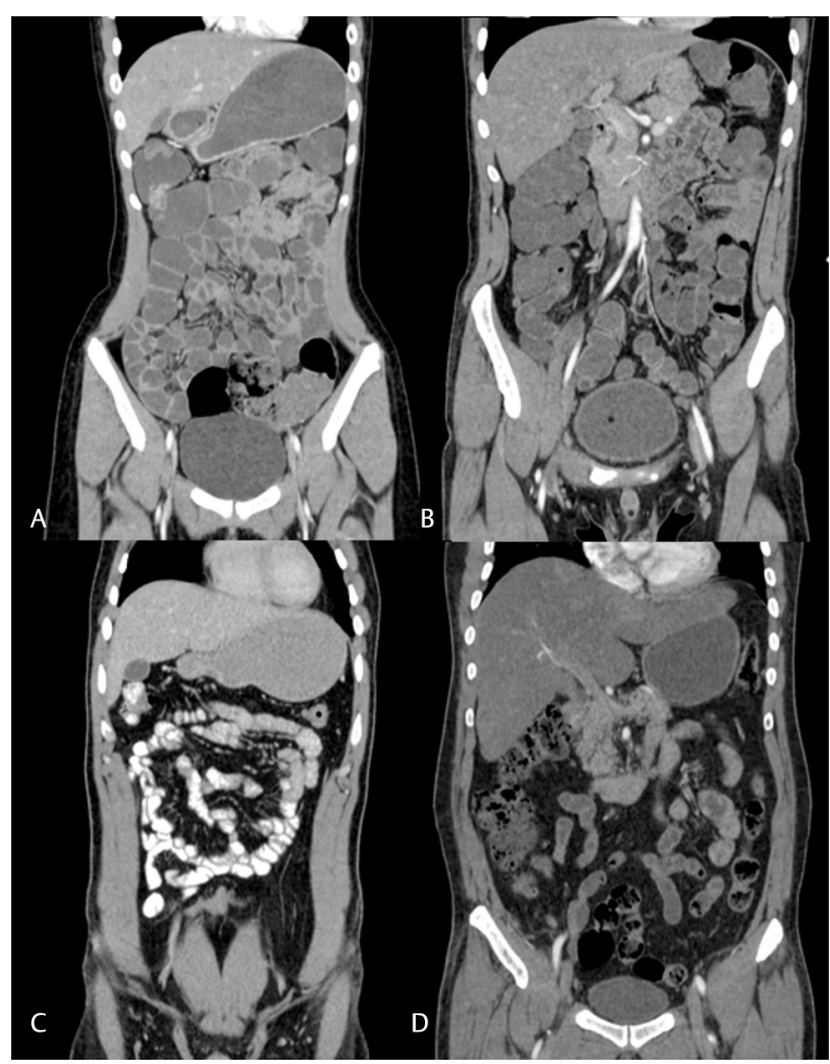

Fig. 5 CT enterography coronal view showing excellent uniform distension, homogeneity, and fold visibility with (A) polyethylene glycol (PEG) as compared with (B) mannitol, (C) iohexol and (D) plain water.

main site of small bowel pathologies. So, an ideal contrast agent should be low attenuating and provide adequate bowel distension and mural detail. All these features are shared by neutral contrast agents. Neutral contrast agents also have limitations that include difficulty in differentiation of extraluminal and intraluminal collections and cystic lesions, but these can be overcome by MPR technique. ${ }^{17,20}$ In our study, we compared PEG, mannitol, iohexol, and plain water. The overall mean distension at second part of duodenum in PEG 
group was $2.42 \pm 0.48 \mathrm{~cm}$, in mannitol group was $2.21 \pm$ $0.34 \mathrm{~cm}$, in iohexol group was $2.18 \pm 0.35 \mathrm{~cm}$, and in plain water group was $1.75 \pm 0.37 \mathrm{~cm}$, at jejunum in PEG group was $2.01 \pm 0.44 \mathrm{~cm}$, in mannitol group was $1.70 \pm 0.17 \mathrm{~cm}$, in iohexol group was $1.45 \pm 0.21 \mathrm{~cm}$, and in plain water group was $1.27 \pm 0.18 \mathrm{~cm}$, at ileum in PEG group was 2.02 $\pm 0.44 \mathrm{~cm}$, in mannitol group was $1.70 \pm 0.17 \mathrm{~cm}$, in iohexol group was $1.49 \pm 0.21 \mathrm{~cm}$, and in plain water group was 1.28 $\pm 0.17 \mathrm{~cm}$, and at ICJ in PEG group was $1.45 \pm 0.30 \mathrm{~cm}$, in mannitol group was $1.06 \pm 0.26 \mathrm{~cm}$, in iohexol group was $0.91 \pm$ $0.21 \mathrm{~cm}$, and in plain water group was $0.81 \pm 0.17 \mathrm{~cm}$. So, the overall distension was better in PEG group followed by mannitol, iohexol and water group.

The fold visibility and homogeneity were also best in PEG group, followed by mannitol, iohexol and water group, which was statistically significant. So, in our study, we found that PEG was the best contrast agent, as it caused maximum distension, better fold visibility and homogeneity in all small bowel parts as compared with other agents. In a study conducted by Zheng, no significant difference was found in bowel distension, wall visibility, and homogeneity among mannitol and PEG group. However, mannitol was superior in terms of tolerability and acceptability as compared with PEG. ${ }^{21}$

Water showed suboptimal distension, especially in distal small bowel, because of its rapid absorption from bowel mucosa. Most of the authors observed that higher the osmolarity better is the distension. ${ }^{17}$ In comparison to neutral contrast agent, positive contrast agent results in obscuration of mucosal enhancement, which is the main feature in some of the small intestinal diseases. Terminal ileum and ICJ is an important part, as it gets involved in majority of small bowel diseases, so distension, fold visibility, and homogeneity at ICJ is important, which was better in PEG group as compared with other groups.

In our study, all the four endoluminal contrast agents were well-tolerated and the palatability was reported best in water group, followed by mannitol group. It was reasonable in iohexol group, and few patients reported bad taste of PEG, which was statistically significant. Nausea was reported in almost 50\% cases of iohexol group, followed by PEG group. Vomiting was reported in two cases in each PEG and iohexol group, which was statistically insignificant. In mannitol group, $27 \%$ of the patients reported mild diarrhea after contrast ingestion, which was statistically significant.

\section{Conclusions}

The following conclusions can be drawn from our study:

- Neutral contrast agents are preferred over positive contrast agents, as mucosal enhancement can be easily detected with neutral contrast agents, which is the main finding in many of small bowel diseases. Out of the three neutral and one positive contrast agents, PEG is a better endoluminal neutral contrast agent that can be used for CTE, as it causes better distension (quantitative and qualitative), fold visibility and homogeneity (qualitative) as compared with mannitol, water and iohexol.
- Distension at ICJ is very important, as it is one of the most common areas involved in small intestinal diseases, and distension was significantly better with PEG as compared with other agents.

- Palatability was poor in few patients with PEG as compared with other groups.

- The result of our study reassured that mannitol causes diarrhea in a few patients.

It is concluded from the above study that PEG is the most suitable contrast agent to carry out CTE. Distension, fold visibility, and homogeneity are the essential features for a better diagnostic outcome of CTE. So, we recommend use of PEG for CTE.

\section{Limitations}

- The number of patients in our study was less, and it was a single centric study. Large-scale and multicentric studies would be required to use PEG as a standard neutral oral contrast agent.

- In our study, we have not included patients of pediatric age group.

- In few cases, the measurement of bowel loop was not possible at specified level, so we have taken measurement below that level.

- We have followed-up the patients for only 1 hour post-CTE to check for any side effects, as it could miss late side effects.

\section{Conflict of Interest}

None declared.

\section{References}

1 Horton KM, Fishman EK. The current status of multidetector row CT and three-dimensional imaging of the small bowel. Radiol Clin North Am 2003;41(2):199-212

2 Wong J, Moore $\mathrm{H}$, Roger $\mathrm{M}$, McKee C. CT enterography: mannitol versus VoLumen. J Med Imaging Radiat Oncol 2016;60(5):593-598

3 Gale HI, Sharatz SM, Taphey M, Bradley WF, Nimkin K, Gee MS. Comparison of $\mathrm{CT}$ enterography and MR enterography imaging features of active Crohn disease in children and adolescents. Pediatr Radiol 2017;47(10):1321-1328

4 Bruining DH, Fletcher JG, Computed tomography enterography. In: Gore RM, Levine MS, eds. Gastrointestinal Radiology. Philadelphia: Saunders; 2015 684-685

5 Ha KH, Park SH, Lee SS, et al. Gastrointestinal tract. In: Hagga JR, Boll DT, eds. CT and MRI of the Whole Body, Philadelphia: Elsevier; 2017 1649-1650

6 Lim BK, Bux SI, Rahmat K, Lam SY, Liew YW. Evaluation of bowel distension and mural visualisation using neutral oral contrast agents for multidetector-row computed tomography. Singapore Med J 2012;53(11):732-736

7 Macari M, Megibow AJ, Balthazar EJ. A pattern approach to the abnormal small bowel: observations at MDCT and CT enterography. AJR Am J Roentgenol 2007;188(5):1344-1355

8 Megibow AJ, BabbJS, Hecht EM, et al. Evaluation of bowel distention and bowel wall appearance by using neutral oral contrast agent for multi-detector row CT. Radiology 2006;238(1):87-95 
9 Shanmuganathan K. Multi-detector row CT imaging of blunt abdominal trauma. Semin Ultrasound CT MR 2004;25(2): 180-204

10 Young BM, Fletcher JG, Booya F, et al. Head-to-head comparison of oral contrast agents for cross-sectional enterography: small bowel distention, timing, and side effects. J Comput Assist Tomogr 2008;32(1):32-38

11 Wong J, Roger M, Moore H. Performance of two neutral oral contrast agents in CT enterography. J Med Imaging Radiat Oncol 2015;59(1):34-38

12 Minordi LM, Vecchioli A, Mirk P, Bonomo L. CT enterography with polyethylene glycol solution vs CT enteroclysis in small bowel disease. Br J Radiol 2011;84(998):112-119

13 Hebert JJ, Taylor AJ, Winter TC, Reichelderfer M, Weichert JP. Low-attenuation oral GI contrast agents in abdominal-pelvic computed tomography. Abdom Imaging 2006;31(1):48-53

14 Thompson SE, Raptopoulos V, Sheiman RL, McNicholas MM, Prassopoulos P. Abdominal helical CT: milk as a low-attenuation oral contrast agent. Radiology 1999;211(3):870-875

15 Allen TL, Mueller MT, Bonk RT, Harker CP, Duffy OH, Stevens $\mathrm{MH}$. Computed tomographic scanning without oral contrast solution for blunt bowel and mesenteric injuries in abdominal trauma. J Trauma 2004;56(2):314-322
16 Lee $\mathrm{CH}$, Haaland B, Earnest A, Tan $\mathrm{CH}$. Use of positive oral contrast agents in abdominopelvic computed tomography for blunt abdominal injury: meta-analysis and systematic review. Eur Radiol 2013;23(9):2513-2521

17 Prakashini K, Kakkar C, Sambhaji C, Shetty CM, Rao VR. Quantitative and qualitative bowel analysis using mannitol, water and iodine-based endoluminal contrast agent on 64-row detector CT. Indian J Radiol Imaging 2013;23(4):373-378

18 Zhang LH,Zhang SZ, Hu HJ, etal. Multi-detector CT enterography with iso-osmotic mannitol as oral contrast for detecting small bowel disease. World J Gastroenterol 2005;11(15):2324-2329

19 Wang YR, Yu XL, Peng ZY. Evaluation of different small bowel contrast agents by multi - detector row CT. Int J Clin Exp Med 2015;8(9):16175-16182

20 Elamparidhi P, Sivaranjanie S, Kumar RR, et al. Comparison of water, mannitol and positive oral contrast for evaluation of bowel by computed tomography. Int J Anat Radiol Surg 2017;6:13-17

21 Zheng MQ, Zeng QS, Yu YQ et al. Evaluation of the performance of two neutral oral contrast agents in computed tomography enterography: a randomized controlled trial. J Dig Dis 2020;21(2):112-119 